\title{
INDENTATION SIZE EFFECT IN HIGH PRESSURE TORSION PROCESSED HIGH ENTROPY ALLOY
}

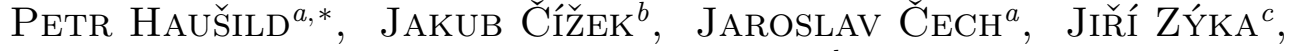 \\ HYOUNG SEOP $\mathrm{KIM}^{d}$ \\ ${ }^{a}$ Czech Technical University in Prague, Faculty of Nuclear Sciences and Physical Engineering, Department of \\ Materials, Trojanova 13, 12000 Praha, Czech Republic \\ ${ }^{b}$ Charles University in Prague, Faculty of Mathematics and Physics, Department of Low Temperature Physics, V \\ Holešovičkách 2, Praha 8, CZ-18000, Czech Republic \\ ${ }^{c}$ UJP PRAHA a.s., Nad Kaminkou 1345, 156 00, Praha, Czech Republic \\ ${ }^{d}$ POSTECH, Department of Materials Science and Engineering, Pohang, 790-784, South Korea \\ * corresponding author: petr.hausild@fjfi.cvut.cz
}

\begin{abstract}
High entropy alloy HfNbTaTiZr in as cast conditions and after high pressure torsion straining was characterized by nanoindentation. The length-scale dependent material response (indentation size effect) was characterized by indentation at various indentation depths. Hardness dependence on the characteristic length (depth of penetration) indicated decomposition of disordered high entropy alloy in the as cast sample, which probably occurred during slow cooling after casting. Subsequent severe plastic deformation by high pressure torsion led on the other hand to the short-range disorder of (originally partially decomposed as cast) structure. Further hardening was generated during high pressure torsion by the mechanisms of grain refinement and increasing dislocation density.
\end{abstract}

KEYWORDS: High entropy alloys, high pressure torsion, indentation size effect, nanoindentation.

\section{INTRODUCTION}

In high entropy alloys (HEAs), the configurational entropy of a multicomponent solid solution phase is maximized so that the Gibbs energy of random solution may be lower than that of possible intermetallic phases. Generally, to achieve high entropy of mixing, the alloys contain typically five or more major elements in equimolar concentrations [1]. The HEAs show promising mechanical properties in applications ranging from (high temperature) structural to biocompatible materials [2, 3].

Mechanical properties of such alloys can further be improved by grain refinement especially by severe plastic deformation. However, studies of ultrafine grained HEAs are rather scarce in the literature. An increase of strength with decreasing grain size was achieved through processing by high pressure torsion (HPT) in the probably most investigated HEA - Cantor alloy i.e., equiatomic $\mathrm{CoCrFeMnNi}$ with face-centered cubic (fcc) structure [4].

Fewer attempts were made to process in such a way HEAs with body-centered cubic (bcc) structure. Recently HfNbTaTiZr bcc HEA was successfully nanostructured by HPT straining [5, 6]. It was reported that grain refinement by HPT resulted in a significant enhancement of the strength of this bcc HEA, keeping excellent ductility during room temperature straining. Nevertheless, there is still a lack of information about the development of microstructure and physical properties of this refractory metal HEA subjected to severe plastic deformation processing.
Recent investigations [5, 6] revealed that thermodynamically stable system of HfNbTaTiZr alloy at room temperature is a mechanical mixture of $\mathrm{Zr}$, Hf rich hcp phase and $\mathrm{Ta}, \mathrm{Nb}$ rich bcc phase. Recrystallization annealing after cold-rolling at $800{ }^{\circ} \mathrm{C}$ led to the transformation of the single-phase bcc material into a two-phase structure composed of bcc $_{1}$ and bcc $_{2}$ phases with lattice parameters $a_{1} \sim 0.3427 \mathrm{~nm}$ and $a_{2} \sim 0.3338 \mathrm{~nm}$, respectively [7].

Chemical analysis revealed that the matrix was slightly enriched in Hf and Zr, while the precipitated second-phase particles were on the other hand slightly enriched in $\mathrm{Ta}$ and $\mathrm{Nb}$. The decomposition of the solid solution (and formation of short range order) after long-term annealing obviously leads to the deterioration of mechanical properties (loss of ductility and decrease of strength). The difference in hardness of both phases is relatively small and both are softer than the random (disordered) solid solution [6]. On the other hand, considerable contribution to the solid solution strengthening can arise from atomic size misfit (phase separation on the nano-meter scale) which is provoked by the high density of vacancies introduced by HPT [5].

The aim of this work was clarification of the relationship between phase (de)composition, microstructure, lattice defects, and length-scale-dependent material response of HfNbTaTiZr HEA in as cast conditions, after solution (disordering) thermal treatment and HPT straining. The length-scale-dependent material response was characterized by indentation at various 


\begin{tabular}{clrrrr}
\hline & As cast & Annealed & $\mathbf{1 / 4}$ turn & 1 turn & $\mathbf{1 5}$ turns \\
\hline$\varepsilon_{\mathrm{pl}}[1]$ & 0 & 0 & 5 & 20 & 300 \\
$\mathrm{H}_{0}[\mathrm{MPa}]$ & 4049 & 4438 & 4947 & 5717 & 5953 \\
$\mathrm{~h}^{*}[\mathrm{~nm}]$ & 45.7 & 76.3 & 49 & 33.5 & 29.3 \\
\hline
\end{tabular}

TABLE 1. List of the samples - equivalent plastic strain $\varepsilon_{\mathrm{pl}}$, identified characteristic lengths $h^{*}$ and hardnesses in the limit of infinite depth $H_{0}$.

indentation depths. The contributions of different mechanisms were attributed to distance between dislocation pinning defects (length scale) and are reported here.

\section{EXPERIMENTAL DETAILS}

\subsection{MATERIAL}

The alloy of equimolar composition HfNbTaTiZr was prepared by vacuum arc melting from pure $\mathrm{Hf}, \mathrm{Nb}$, $\mathrm{Ta}$, Ti and $\mathrm{Zr}$ metals and cast into water cooled $\mathrm{Cu}$ crucible. In order to ensure proper mixing, the ingot was flipped and remelted eight times. Disc of $20 \mathrm{~mm}$ diameter and $1 \mathrm{~mm}$ thickness were HPT strained at room temperature and pressure of $2.5 \mathrm{GPa}$. Straining was performed to $1 / 4$ turn, 1 turn and 15 turns of the piston (see Fig. 1). Corresponding equivalent plastic strains $\varepsilon_{\mathrm{pl}}$ (at the characterized areas) are 5, 20 and 300 (see Table 1). More details can be found in Ref. [5]. One non-deformed (as cast) sample was also annealed for 2 mins at $1600{ }^{\circ} \mathrm{C}$ (followed by rapid cooling) for reasons described in section Results and discussion.

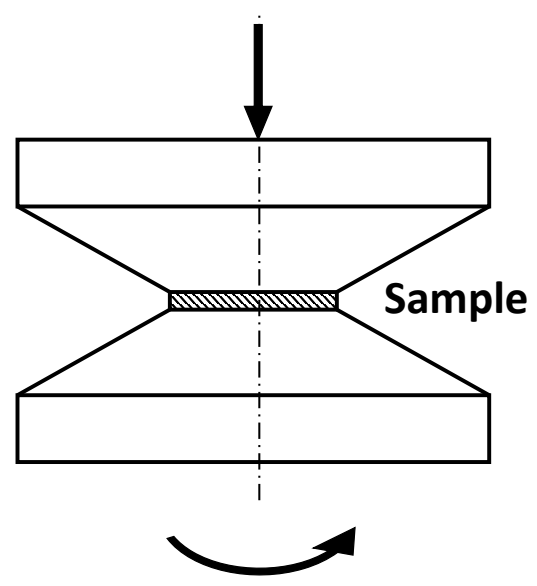

Figure 1. Scheme of high pressure torsion straining.

\subsection{NANOINDENTATION}

Surface of samples was polished by standard metallographical procedure with final step made using $0.04 \mu \mathrm{m}$ colloidal silica to avoid the surface layer affected by mechanical grinding and polishing. Nanoindentation measurements were performed using an Anton Paar $\mathrm{NHT}^{2}$ Nanoindentation Tester with
Berkovich diamond indenter using instrumented indentation technique [8, 9].

In order to obtain the mechanical response from different depth, so called continuous multi cycle (CMC) indentations with increasing load were performed varying the maximum load at each cycle from $1 \mathrm{mN}$ up to $500 \mathrm{mN}$. This type of load cycle allows characterization at different load (depth) levels at the same position (see e.g. Refs. [10, 11]). Loading time was 10 s per each cycle for CMC, followed by $5 \mathrm{~s}$ hold at the maximum load and unloading time $10 \mathrm{~s}$ per each cycle. The results (indentation hardness) were evaluated according to the ISO 14577 standard. The indentations were made on sample surface in the position corresponding to the half radius, i.e. in the location of most homogeneously distributed deformation after HPT straining. At least $20 \mathrm{CMC}$ indentations were performed for each material state (deformation condition).

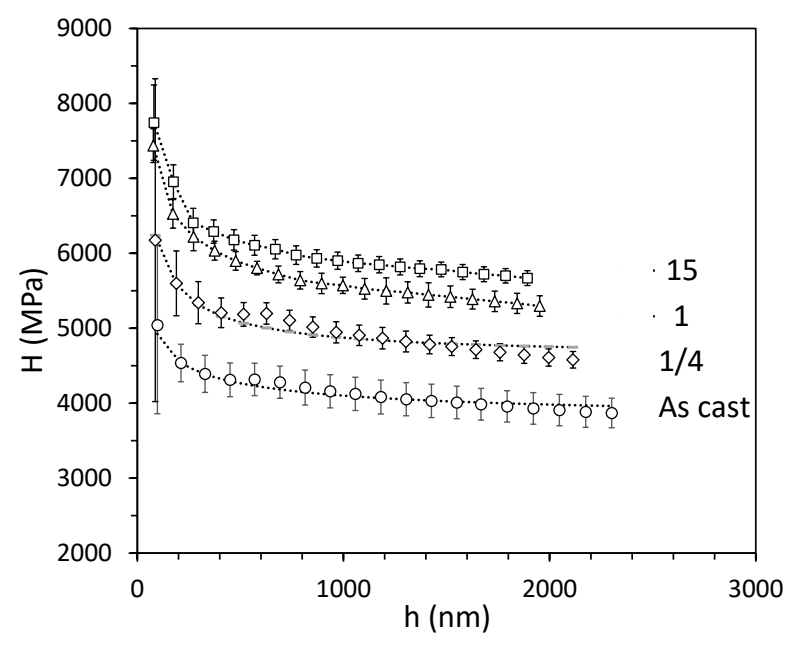

FigURE 2. Indentation size effect in as cast sample and samples HPT strained to $1 / 4$ turn, 1 turn and 15 turns.

\section{Results And Discussion}

Results of hardness measurements as a function of penetration depth for as cast sample and samples HPT strained from $1 / 4$ turn to 15 turns are shown in Fig. 2. It can be seen in this figure that hardness increases with increasing strain and that all samples show strong indentation size effect in sub-micron penetration depths. Using Nix nad Gao approach [12] based on the geometrically necessary dislocations, the 
depth dependence of the hardness $H$ can be related to the characteristic length $h^{*}$ through the relation:

$$
\frac{H}{H_{0}}=\sqrt{1+\frac{h^{*}}{h}}
$$

where $h$ is a given depth of penetration, and $H_{0}$ is the hardness in the limit of infinite depth $(\sim$ macroscopic hardness).

Depth dependence of the hardness is plotted according to Eq. 1 for different levels of straining in Fig. 3 and the parameters $H_{0}$ and $h^{*}$ obtained by least square fit are listed in Table 1

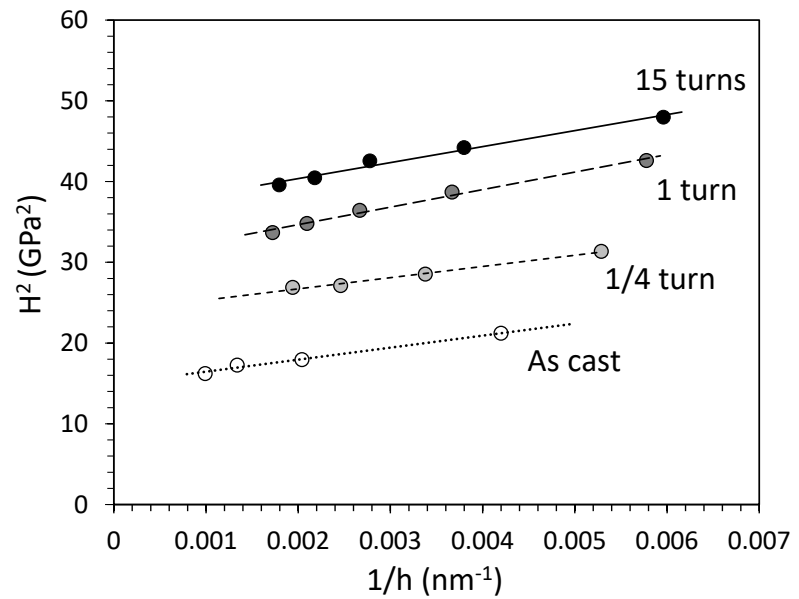

Figure 3. Depth dependence of the hardness plotted for as cast sample and samples HPT strained from 1/4 turn to 15 turns according to Eq. 1 .

As can be expected, the hardness in the limit of infinite depth $H_{0}$ is increasing with the increasing level of HPT straining (HPT leads to the strain hardening [13). On the other hand, the characteristic length $h^{*}$ is decreasing with the increasing level of deformation. It is to note that the values of characteristic lengths $h^{*}$ are quite low, which indicates fine microstructural features involved in the dislocation pinning process.

According to Nix and Gao 12, the characteristic length $h^{*}$ depends on the hardness in the limit of infinite depth $H_{0}$ as follows:

$$
h^{*} \sim \frac{1}{H_{0}^{2}}
$$

Fig. 4 shows that characteristic depth $h^{*}$ dependence on the hardness in the limit of infinite depth $H_{0}$ fulfils relation (2) for HPT deformed samples, but the as-cast sample clearly does not follow this relation. As mentioned in the Introduction, the anomalous behavior of the as cast sample is likely caused by the decomposition of disordered (high entropy) bcc solid solution into two $\mathrm{bcc}_{1}$ and $\mathrm{bcc}_{2}$ phases.

Such short-range atomic redistribution is hard to be characterized by conventional techniques such $\mathrm{X}$-ray diffraction or transmission electron microscopy as the variation of lattice parameter is very small

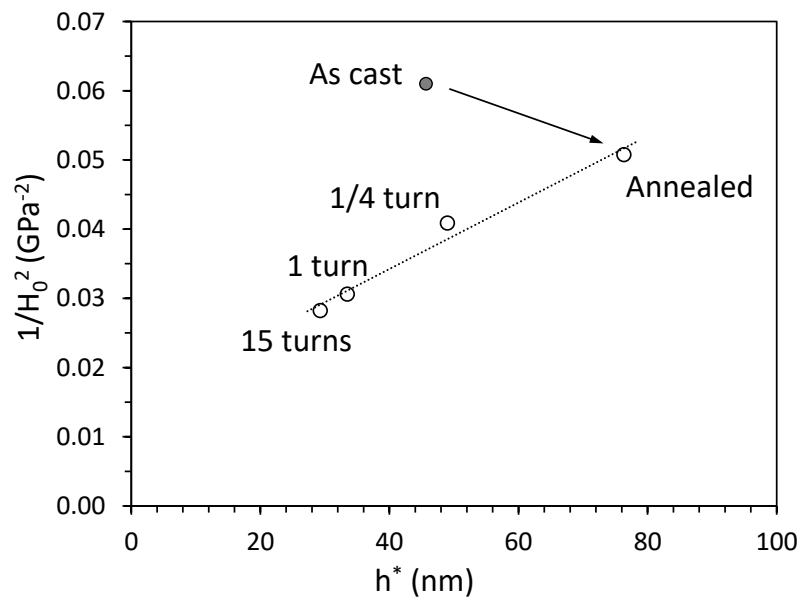

Figure 4. Characteristic depth dependence of the hardness in the limit of infinite depth for as cast sample, annealed sample and samples HPT strained from $1 / 4$ turn to 15 turns.

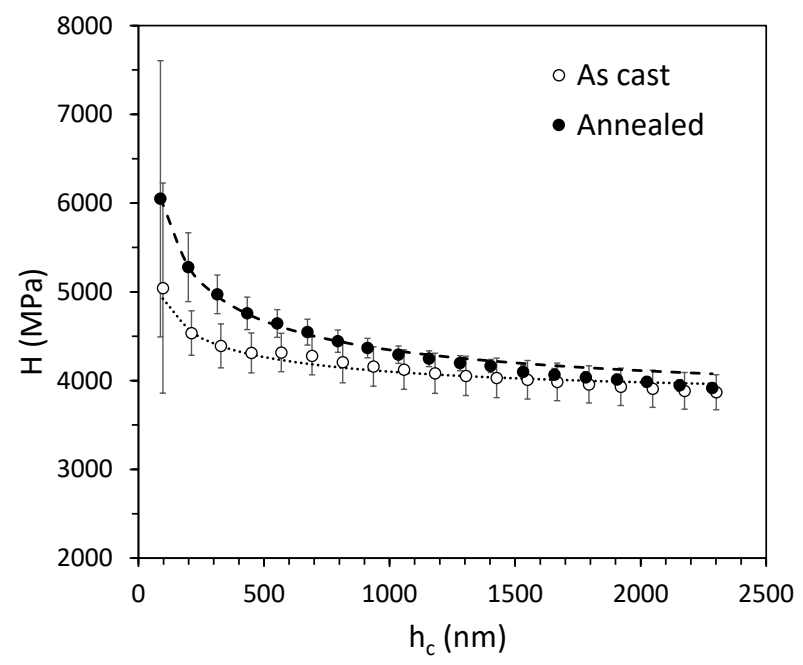

Figure 5. Comparison of depth dependence of the hardness in the as cast and annealed samples.

and the size of domains is in the order of tens of $\mathrm{nm}$ (see parameter $h^{*}$ ). Therefore, the as cast sample was annealed at (high) temperature of $1600{ }^{\circ} \mathrm{C}$ with subsequent rapid cooling in order to preserve the random solid solution and subsequently characterized by nanoindentation.

The results of hardness measurements in as cast and annealed state are compared in Fig. 5 . As can be seen in Table 1, the parameter $h^{*}$ significantly increased after annealing, which is consistent with the hypothesis of short-range order disappearance. The tendency given by the relation (Eq. 2 ) is now in good agreement for both annealed and HPT strained samples (Fig. 4). Thus, similarly to high temperature annealing the HPT led to the short-range disorder of (originally partially decomposed) as-cast sample (severe plastic deformation leads to an extension of solid solubility [14]). Subsequent hardening in 
HPT strained samples is related rather to grain (cell) refinement and increasing dislocation density as observed in Ref. [5] using transmission electron microscopy.

\section{Conclusions}

The results of the present study can be summarized as follows:

- High pressure torsion led to a significant (and progressive) hardening of high entropy HfNbTaTiZr alloy.

- Both as cast and HPT strained samples showed length-scale dependent material response (indentation size effect).

- As cast sample presented different dependence of characteristic length $h^{*}$ on the hardness in the limit of infinite depth $H_{0}$ than HPT deformed and annealed samples, which indicates short-range decomposition of disordered high entropy alloy.

- It can be concluded that severe plastic deformation by HPT produced the short-range disorder of originally partially decomposed as cast structure, which was followed by hardening due to the grain refinement and increasing dislocation density intorduced by HPT process.

- Annealing of as cast sample at $1600{ }^{\circ} \mathrm{C}$ with subsequent rapid cooling in order to stabilize the disordered structure led to the same dependence of characteristic length on the hardness in the limit of infinite depth as in the case of HPT strained samples.

\section{ACKNOWLEDGEMENTS}

This research was carried out in the frame of the projects CZ.02.1.01/0.0/0.0/16_019/0000778 (Centre of Advanced Applied Sciences) and 17-17016S (Czech Science Foundation).

\section{REFERENCES}

[1] M.-H. Tsai, J.-W. Yeh. High-entropy alloys: A critical review. Materials Research Letters 2(3):107-123, 2014. DOI:10.1080/21663831.2014.912690

[2] D. Miracle, O. Senkov. A critical review of high entropy alloys and related concepts. Acta Mater 122:448 - 511, 2017. DOI:10.1016/j.actamat.2016.08.081.

[3] J. Chen, X. Zhou, W. Wang, et al. A review on fundamental of high entropy alloys with promising high-temperature properties. J Alloy Comp 760:15 30, 2018. DOI:10.1016/j.jallcom.2018.05.067.
[4] A. Heczel, M. Kawasaki, J. Lábár, et al. Defect structure and hardness in nanocrystalline $\mathrm{CoCrFeMnNi}$ high-entropy alloy processed by high-pressure torsion. $J$ Alloy Comp 711:143-154, 2017. DOI:10.1016/j.jallcom.2017.03.352.

[5] J. Čížek, P. Haušild, M. Cieslar, et al. Strength enhancement of high entropy alloy HfNbTaTiZr by severe plastic deformation. J Alloy Comp 768:924-937, 2018. DOI:10.1016/j.jallcom.2018.07.319

[6] B. Schuh, B. Völker, J. Todt, et al. Thermodynamic instability of a nanocrystalline, single-phase TiZrNbHfTa alloy and its impact on the mechanical properties. Acta Mater 142:201-212, 2018. DOI:10.1016/j.actamat.2017.09.035

[7] O. N. Senkov, S. L. Semiatin. Microstructure and properties of a refractory high-entropy alloy after cold working. J Alloy Comp 649:1110-1123, 2015. DOI:10.1016/j.jallcom.2015.07.209.

[8] W. Oliver, G. Pharr. An Improved Technique for Determining Hardness and Elastic-Modulus Using Load and Displacement Sensing Indentation Experiments. $J$ Mater Res 7(6):1564-1583, 1992. DOI:10.1557/JMR.1992.1564

[9] W. Oliver, G. Pharr. Measurement of hardness and elastic modulus by instrumented indentation: Advances in understanding and refinements to methodology. $J$ Mater Res 19(1):3-20, 2004. DOI:10.1557/jmr.2004.0002

[10] P. Haušild, A. Materna, L. Kocmanová, J. Matějíček. Determination of the individual phase properties from the measured grid indentation data. J Mater Res 31(22):3538-3548, 2016. DOI:10.1557/jmr.2016.375

[11] P. Haušild, J. Čech, A. Materna, J. Matějíček. Statistical treatment of grid indentation considering the effect of the interface and the microstructural length scale. Mech Mater 129:99 - 103, 2019. DOI:10.1016/j.mechmat.2018.11.006.

[12] W. Nix, H. Gao. Indentation size effects in crystalline materials: A law for strain gradient plasticity. J Mech Phys Solids 46(3):411-425, 1998. DOI:10.1016/S0022-5096(97)00086-0

[13] A. P. Zhilyaev, T. G. Langdon. Using high-pressure torsion for metal processing: Fundamentals and applications. Progress in Mater Sci 53(6):893 - 979, 2008. DOI:10.1016/j.pmatsci.2008.03.002

[14] O. Senkov, F. Froes, V. Stolyarov, et al. Microstructure of aluminum-iron alloys subjected to severe plastic deformation. Scripta Mater 38(10):1511 1516, 1998. DOI:.10.1016/S1359-6462(98)00073-6 